\title{
Determination of the atomic structure of scanning probe microscopy tungsten tips by field ion microscopy
}

\author{
Anne-Sophie Lucier, Henrik Mortensen, Yan Sun, and Peter Grütter* \\ Department of Physics, McGill University, 3600 University Street, Montréal, Québec, Canada H3A 2T8
}

(Received 19 September 2005; published 19 December 2005)

\begin{abstract}
Detailed knowledge of the tip apex structure is necessary for quantitative comparison between theory-based simulations and experimental observations of tip-substrate interactions in scanning probe microscopy (SPM). Here, we discuss field ion microscopy (FIM) techniques to characterize and atomically define SPM tungsten tips. The tip radius can be estimated from field emission data, while FIM imaging allows the full atomic characterization of the tip apex. We find that when FIM is applied to tips with a radius of a few nanometers (as is desirable for high-resolution atomic force microscopy imaging), limitations not apparent with less sharp tips arise; successful resolution of these limitations will extend the utility of FIM. Field evaporation can be used to atomically engineer the apex into a desired atomic configuration. Starting from a W(111) wire, a tip terminating in three atoms can reproducibly be fabricated; due to its geometry and stability, this apex configuration is well suited for application as an atomically defined electrical contact in SPM experiments aimed at understanding contact mechanics at the atomic scale.
\end{abstract}

DOI: $10.1103 /$ PhysRevB.72.235420

PACS number(s): $68.37 . \mathrm{Vj}, 39.25 .+\mathrm{k}$

\section{INTRODUCTION}

Quantitative simulations of scanning probe microscopy (SPM) experiments usually need to make an assumption about the atomic tip structure. Differences in tip structure or chemistry are, however, predicted to lead to major differences. ${ }^{1}$ Experimentally, it is a well-known fact that "optimal" tips exist for high-resolution imaging, spectroscopy, and manipulation. These optimal tips can differ for different applications and are seldom, if ever, characterized experimentally. In the field of high-resolution atomic force microscopy (AFM), the mesoscopic tip radius plays a key role in enabling atomic scale imaging, as the mesoscopic range forces (such as van der Waals or electrostatic interactions) can mask the short-range chemical forces leading to atomic scale imaging. Dissipation contrast with atomic scale spatial variation in noncontact AFM often abruptly changes magnitude (and when imaging multiatom samples such as $\mathrm{KBr}$ sometimes even inverts contrast) - a major conundrum in this field. ${ }^{2,3}$ It is accepted that an important source of contrast is due to the atomic tip structure-controlling this might allow the quantitative understanding and possibly even the exploitation of dissipation contrast in chemical identification by noncontact AFM of atomic species. SPM configurations are being used in the field of molecular electronics ${ }^{4}$ where one of the major obstacles in quantitative understanding of the relationship between molecular structure and electronic properties (as well as issues of reproducibility) is due to a lack of detailed characterization of the contact formed by the SPM tip. Finally, the field of nanotribology, which attempts to understand the mechanical properties of a model asperity and a surface, needs the model asperity to be characterized atomically in order to compare quantitatively to state of the art simulations.

Field ion microscopy (FIM) is a more than 50-year-old technique $^{5}$ that has been successfully used to characterize SPM tips. ${ }^{6}$ Fink demonstrated more than ten years ago that
FIM could also be used to machine structures atom by atom. ${ }^{7}$ We have used such atomically machined FIM tips in a combined ultrahigh-vaccum (UHV) AFM-scanning tunneling microscopy (STM) system to investigate the formation of mechanical and electrical contacts under well-defined conditions. ${ }^{8-10}$ Unfortunately, despite the obvious advantages and potential for solving many fundamental issues in different fields, combined FIM and SPM systems are rare due to the complexity and challenges of combining these two techniques. ${ }^{6,8,11-14}$ In this paper, we describe in detail the preparation, characterization, and fabrication by FIM of atomically defined $\mathrm{W}(111)$ tips.

We chose $\mathrm{W}$ for detailed studies, although we have achieved similar results with Ir and Ni tips. The advantage of $\mathrm{W}$ tips is that they are relatively easy to prepare and are stiff enough to withstand the mechanical stress imposed by the high electrical fields during FIM imaging. Since the W tip will be used for STM imaging as well as for probing electrical properties of the substrate, the main requirements for this electrode are the following: it should be very sharp and free of contamination, it should have an atomically stable apex and it should be fully structurally characterized before being brought into contact with the substrate. A tip fabrication technique satisfying these requirements has been developed and several tip characterization tools have been explored; the various results obtained on polycrystalline $\mathrm{W}$ tips and on single-crystal, (111)-oriented W tips are presented throughout this paper.

Typical FIM applications involve studies of surface phenomena and thus require the use of dull tips with large, open crystallographic facets; when very sharp tips are observed under FIM, important limitations arise due to the nature of the microscope which can become problematic in the interpretation of the FIM images and thus the atomic characterization of the tips. This paper will also emphasize the limitations of FIM when applied to STM tips and the need for solutions which would dramatically extend FIM's utility. 


\section{EXPERIMENTS, RESULTS, AND DISCUSSION}

\section{A. Tip preparation}

To prepare our SPM tips, we use the well-known electrochemical etching method. ${ }^{5,15-19}$ Our $\mathrm{W}$ tips are made from either a $0.1-\mathrm{mm}$-diameter polycrystalline wire or a 0.13 -mm-diameter single-crystal, (111)-oriented wire. To perform the electrochemical etching we use a freshly prepared $7.5 \mathrm{~mol} / 1 \mathrm{KOH}$ solution. We set the immersed length of the $\mathrm{W}$ wire to be $0.5 \mathrm{~mm}$, and to generate the etching reaction, we use a bias voltage of $3.0 \mathrm{~V}_{d c}$ with respect to a counterelectrode made with a loop of steel located at the bottom of the solution container.

The etching happens at the air/liquid interface, where a meniscus of solution is formed around the tip; since the reaction rate is a lot slower at the top of the meniscus than at the bottom, a necking effect occurs, which eventually results in the lower portion of the wire breaking off. ${ }^{20}$ The tip is sharpest at the moment of dropoff, ${ }^{20-23}$ so the tip and the counterelectrode are connected to a controller which monitors the current between the anode and the cathode in order to quickly switch off the voltage when a sudden drop in current is detected. The whole etching operation requires a few minutes.

The tip is cleaned immediately by carefully dipping it for a few seconds in distilled water and rinsing it with acetone and ethanol. The purpose of this step is to remove residues left by the etching process. The tip is then gently dried with clean, compressed nitrogen gas.

W tips resulting from a successful electrochemical etching process are quickly transferred to the preparation chamber of our UHV system ${ }^{11,24}$ for a further cleaning step, as their surface is inevitably covered with contaminants which cannot be removed by mere rinsing. It is well known that an oxide layer (mainly $\mathrm{WO}_{3}$ ) develops on the tip shank during electrochemical etching. ${ }^{25,26}$ Auger microscopy and energy dispersive x-ray analysis have also revealed the presence of other surface contaminants such as carbon, nitrogen, alkalimetal hydroxides (e.g., $\mathrm{KOH}$ ) and microcrystals (e.g., $\left.\mathrm{WO}_{3} \mathrm{~K}\right)$, which are left on the tip from the electrochemical bath and exposure to the atmosphere. ${ }^{27-30}$

Heating the tip at high temperatures in UHV is a very efficient way to desorb the contamination layer. At temperatures above $1000 \mathrm{~K}$, the following reaction takes place: ${ }^{31,32}$

$$
2 \mathrm{WO}_{3}+\mathrm{W} \rightarrow 3 \mathrm{WO}_{2} \uparrow .
$$

From Eq. (1), it can be seen that the tungsten trioxide covering the tip will react with $\mathrm{W}$ to form tungsten dioxide; the latter is volatile and sublimates around $1075 \mathrm{~K} .{ }^{31,33} \mathrm{~A}$ clean metallic W surface is therefore left behind. ${ }^{28,29,31}$ Another advantage of this annealing process is that it heals the crystallographic defects generated by the electrochemical etching and the dropoff, and it smooths out the tip surface. ${ }^{34-36}$

Our tips are annealed by resistively heating them to high temperatures. This is achieved by running a controllable current through a conducting wire in contact with the tip. After trying various annealing temperatures and times, we found that the method yielding the best results was to first flash anneal the tip for $2 \mathrm{~s}$ to a bright orange glow, which would usually correspond to a current between 3.0 and $4.5 \mathrm{~A}$, and then to lower the current to about $2.0 \mathrm{~A}$ for at least $5 \mathrm{~min}$. The goal of the first annealing step-what we called flash annealing - is to go to a high enough temperature to remove the oxide layer, but not too high nor for too long to induce substantial blunting of the tip. It has been reported that at around $900 \mathrm{~K}$ the tip starts to glow ${ }^{37}$ and thus exhibits a dark red color; between 1175 and $1300 \mathrm{~K}$ the tip has an orange glow; ${ }^{11,34}$ and around $1375 \mathrm{~K}$ the tip has a yellow glow. ${ }^{11}$ Perception of colors is a subjective matter, but we can deduce that our bright orange glow probably corresponds to a temperature close to $1300 \mathrm{~K}$. The second annealing step, which consists in heating the tip at a much lower temperature for a longer time, is intended to allow other remaining impurities to desorb and also to degas the tip holder.

\section{B. Characterization of the tips by field emission analysis}

If a negative potential is applied to a sharp tip, it will field emit a measurable current at a few hundred volts. Monitoring the field emission characteristics of the tip is a quick way to verify its sharpness and a more involved examination of the field emission data can even lead to the extraction of a tip radius value. After annealing the tip in the preparation chamber, an oxygen-free copper plate is brought to within about $2.5 \mathrm{~cm}$ of the tip; this plate serves as the anode collecting the field emitted electrons.

Fowler and Nordheim studied field emission extensively and derived the following expression, which relates the emitted current density $i$ to the electrostatic field $F$ at the metallic surface: ${ }^{38,39}$

$$
i=6.2 \times 10^{-6} \frac{(\mu / \phi)^{1 / 2}}{\alpha^{2}(\mu+\phi)} F^{2} \times \exp \left(-6.8 \times 10^{7} \frac{\phi^{3 / 2} \alpha}{F}\right),
$$

where $\phi$ is the metal's work function in $\mathrm{eV}, \mu$ is the Fermi level in $\mathrm{eV}, \alpha$ is the image correction factor accounting for the changes in field emission caused by the Coulombic interaction of the tunneling electron and its induced image charge on the metal surface, $i$ is in $\mathrm{A} / \mathrm{cm}^{2}$, and $F$ is in $\mathrm{V} / \mathrm{cm}$. In the case of a tip, the field is strongest at the apex and can be estimated by modeling the tip as a sphere buried in a conical shank, giving for this field the following expression:

$$
F=\frac{V}{k R},
$$

where $V$ is the applied voltage, $R$ is the tip radius, and $k$ is the field reduction factor which is due to the presence of a shank. ${ }^{39}$ From Eq. (3), one can easily see that for a given applied voltage and field reduction factor, a smaller tip apex radius will yield a higher electrostatic field; we also know from Eq. (2) that a higher electrostatic field will produce a higher field emission current density. Multiplying Eq. (2) by the total field emitting area $a$ (in $\mathrm{cm}^{2}$ ) and using Eq. (3) to express the electrostatic field $F$, we obtain the total field emitted current $I$ (in $\mathrm{A}$ ) as a function of the tip radius $R$ (in $\mathrm{cm})$ : 


$$
\begin{aligned}
I= & a 6.2 \times 10^{-6} \frac{(\mu / \phi)^{1 / 2}}{\alpha^{2}(\mu+\phi)} \frac{V^{2}}{(k R)^{2}} \\
& \times \exp \left(-6.8 \times 10^{7} \frac{\phi^{3 / 2} \alpha k R}{V}\right) .
\end{aligned}
$$

Equation (4) provides the explicit description of the relationship between tip sharpness and field emission data: for any tip voltage, the smaller the tip apex radius, the higher the total field emitted current.

Estimating the tip radius can be done through a direct application of the Fowler-Nordheim theory on field emission. Dividing Eq. (4) by $V^{2}$ and taking the natural logarithm on both sides, we obtain

$$
\begin{aligned}
\ln \frac{I}{V^{2}}= & \ln \left(a 6.2 \times 10^{-6} \frac{(\mu / \phi)^{1 / 2}}{\alpha^{2}(\mu+\phi)(k R)^{2}}\right) \\
& -6.8 \times 10^{7} \frac{\phi^{3 / 2} \alpha k R}{V}
\end{aligned}
$$

where $I$ is in $\mathrm{A}, V$ in $\mathrm{V}, a$ in $\mathrm{cm}^{2}, \mu$ and $\phi$ in $\mathrm{eV}$, and $R$ in $\mathrm{cm}$. From Eq. (5), it can be seen that a plot of $\ln \left(I / V^{2}\right)$ versus $1 / V$ will yield a straight line of slope $-6.8 \times 10^{7} \phi^{3 / 2} \alpha k R$. This particular way of graphing field emission data is called a Fowler-Nordheim (FN) plot and simply requires one to record the total field emission current for various applied voltages. Provided that the values of $\phi, \alpha$, and $k$ are known, it is a straightforward matter to evaluate the tip radius from the slope of a FN plot. ${ }^{35,37,39-41}$

We obtained several FN plots from our field emission measurements on polycrystalline $\mathrm{W}$ tips, but before showing these results and attempting to extract a tip radius value it is important to discuss the choice of appropriate values for $\phi$, $\alpha$, and $k$.

The work function $\phi$ of a particular material displays crystallographic anisotropy. By definition, a polycrystalline material is composed of differently oriented crystallites; however, the fabrication process yielding polycrystalline wire results in a preferred grain orientation along the wire axis. ${ }^{39}$ For body-centered cubic (bcc) metals like W, the favored orientation is (110), ${ }^{15,39}$ this has been confirmed for all our polycrystalline tips by FIM measurements. Since the topmost layer of atoms on the apex of polycrystalline tips belongs to the (110) plane, it would be tempting to use the work function value corresponding to this particular plane in the analysis of our FN plots, as was done by some authors. ${ }^{40}$ However, because the tip apex is a spheroidal-looking surface composed of an arrangement of variously oriented flat facets, electrons will be field emitted from several crystallographic planes and not just from the plane perpendicular to the tip axis. Moreover, in the case of W, the (110) plane happens to have the highest work function $\left[\phi_{110}=5.25 \mathrm{eV}\right.$ (Ref. 42)] and thus the lowest emission of all planes, ${ }^{39}$ this enforces the idea that other adjacent planes with a lower work function [for example, $\phi_{100}=4.63 \mathrm{eV}$ and $\phi_{111}$ $=4.47 \mathrm{eV}$ (Ref. 42)] will contribute in great proportion to the total field emitted current. Therefore, using the average work function for $\mathrm{W}\left[\phi_{W}=4.5 \mathrm{eV}\right.$ (Ref. 33)], which is heavily

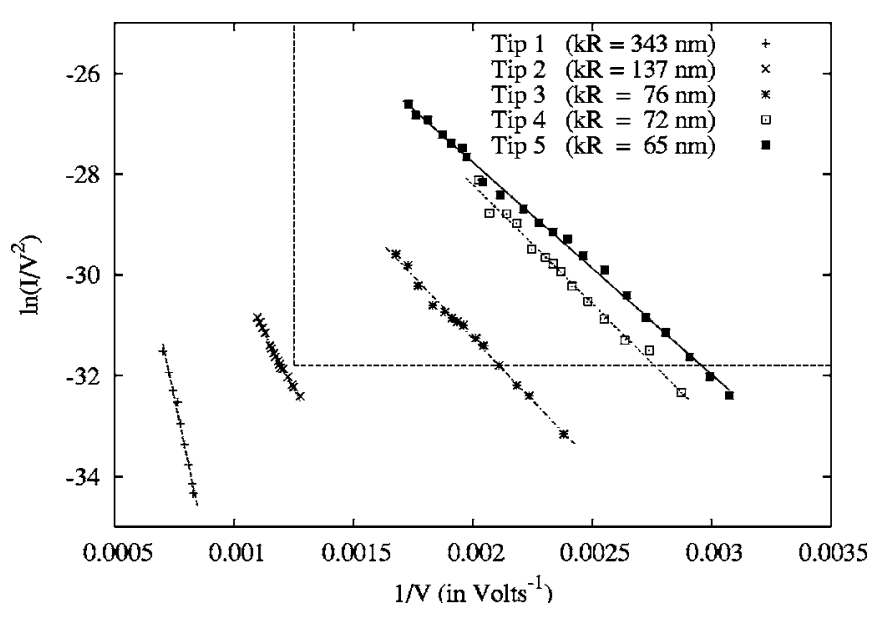

FIG. 1. FN plots corresponding to five different polycrystalline W tips. A smaller slope and a displacement of the plot from left to right are the signature of a sharper tip. The dashed lines illustrate our empirical test for tip sharpness, which is explained in the text.

weighted in favor of the strongly emitting planes, ${ }^{39}$ as an estimate of the overall work function of our tip apexes seems quite reasonable.

It is common practice to take the image correction factor $\alpha$ to be unity when trying to evaluate the tip radius from $\mathrm{FN}$ plots, ${ }^{35,39}$ which is equivalent to ignoring the effect of the image potential. This is acceptable for two reasons. First, in most field emission applications, $\alpha$ actually turns out to be close to unity; ${ }^{39}$ second, only a rough estimate of the tip radius can be obtained from a $\mathrm{FN}$ plot since an accurate determination of the values of $\phi, \alpha$, and $k$ depends on the exact tip apex geometry, which is unknown when field emission measurements are performed.

The field reduction factor $k$ is the hardest parameter to estimate, since, for very sharp tips in particular, its value is highly related to the tip shape and apex radius. A systematic study of field emission characteristics along with electron microscopy imaging of several tips has been done and reveals that a value of $k \sim 5$ is adequate for most geometries encountered in practice. ${ }^{39}$ Other experiments indicated that $k$ is usually between 5 and 8 for slender tips, and its value can drop to about 3 when the tip is annealed because of the smooth bulbous apex resulting from blunting effects. ${ }^{15}$ Therefore, from field emission measurements alone, only a range of possible radius values can be found for a tip.

Figure 1 presents field emission data plotted for five different polycrystalline $\mathrm{W}$ tips. We can easily differentiate between the sharper tips and the blunter tips simply by looking at the slope. We know from Eq. (5) that a smaller slope is characteristic of a sharper emitter; a shift of the plot to higher values of $1 / V$ (from left to right in Fig. 1) is also a clear indication of a sharp tip since this means that a current is emitted for lower applied voltages. ${ }^{29,40,43}$ These two phenomena are nicely combined in Fig. 1; it can be seen that the slope indeed decreases as the plot is shifted to the right. The leftmost curve thus corresponds to the largest tip radius, and the rightmost curve to the sharpest tip. The $k R$ values extracted from the linear fits are given in the graph legend to allow for an easier comparison between the tips. An analysis 
of FN plots is very powerful in that it allows a useful comparison between the sharpness of tips, which, due to consistency in preparation, we can assume to have similar geometry and thus a similar $k$ value. Two of the tips presented in Fig. 1 have been observed under a scanning electron microscope and their radius has been measured directly from the obtained images; tip 2, with a $k R$ value of $137 \mathrm{~nm}$, had a radius of $\sim 40 \mathrm{~nm}$ whereas tip 5, with a $k R$ value of $65 \mathrm{~nm}$, had a radius of $\sim 20 \mathrm{~nm}$. One can readily notice that tip 2, with a $k R$ value about twice as large as that of tip 5, also had a radius twice as large as that of tip 5. That the radii seem to scale proportionally to the $k R$ values supports the assumption that $k$ is constant for our tips; this $k$ value can then be used to get a good estimate of the radius of our tips from our FN plots alone. Here we obtain $k \sim 3.4$ for tip 2 and $k \sim 3.3$ for tip 5; this falls in the range of typical $k$ values listed in the previous paragraph.

FN plots also provide us with a convenient way of discarding tips that are not sharp enough to be imaged by our FIM setup. The maximum positive voltage that can be applied to our FIM tips is $7 \mathrm{kV}$ because of the breakdown voltage of the insulation on the wire leading to the tip. A higher-rated cable would be significantly thicker and rigid, which would increase the vibration coupling to the tip; this would be detrimental when using the tip in STM mode. By Eq. (3), one can relate the best imaging voltage $\left(V_{\mathrm{BI}}\right)$ of the tip to the best imaging field $\left(F_{\mathrm{BI}}\right)$ of the FIM imaging gas (helium in our case) in the following manner:

$$
F_{\mathrm{BI}}=\frac{V_{\mathrm{BI}}}{k R} \text {. }
$$

Knowing that $F_{\mathrm{BI}}$ for helium is $44 \mathrm{~V} / \mathrm{nm}$ and that our highest available $V_{\mathrm{BI}}$ is $7 \mathrm{kV}$, then Eq. (6) predicts that our tips have to meet the condition $k R<160 \mathrm{~nm}$ in order to be clearly imaged with our FIM. This information is readily available in the FN plots. Applying this selection criterion to the FN plots shown in Fig. 1, we see that only tip 1 should be discarded.

In practice though, we have found empirically that the tips which could emit a current of about $10 \mathrm{nA}$ for an applied negative voltage smaller than $800 \mathrm{~V}$ were suitable candidates for FIM studies in our particular setup. This quick test, although not yielding a quantitative description of the tip sharpness, is a very useful diagnostic in determining whether a tip is sharp enough for FIM before actually trying to image it with FIM. For the tips that satisfy this requirement, it is then worth acquiring their FN plot in order to establish their actual radius. This empirical selection criterion can be applied to Fig. 1 in the following manner: if the FN plot of a tip (or its extrapolation) crosses the top right area of the graph delimited by the dashed lines, then that tip is sharp enough to be imaged by our FIM. Following this criterion, tips 1 and 2 should be discarded, whereas tips 3, 4, and 5 should be kept for FIM imaging. Note that tip 2 is a borderline case and a sharp image of its apex would be difficult to obtain with our microscope; it should thus be discarded, even if its $k R$ value of $137 \mathrm{~nm}$ is below $160 \mathrm{~nm}$. One can see that the two selection criteria are in good agreement for tips which should clearly be kept or discarded. However, for tips with a $k R$ value close to $160 \mathrm{~nm}$, our empirical findings should obviously prevail, since the criterion obtained from Eq. (6) only provides a rough guideline for tip selection.

An interesting effect that was reported before ${ }^{34}$ has been observed while we were testing our tips for sharpness. For tips that were not quite sharp enough to be imaged with our FIM, we would apply a higher bias in order to extract field emission currents up to $5 \mu \mathrm{A}$ for a few seconds and then decrease the bias. This extra treatment, sometimes after several repetitions, would often allow us to lower the voltage required to reach $10 \mathrm{nA}$ by up to $200 \mathrm{~V}$. Some of these tips would become sharp enough to be imaged with our FIM, and a FN plot would then be constructed to find their radius. There are a few possible explanations to this sudden apparent increase in tip sharpness. A hypothesis is that drawing such high currents could result in further cleaning of the tips. One can imagine that a current of $5 \mu \mathrm{A}$ flowing through the tip could yield an apex temperature high enough to sublimate some of the tungsten oxide that might be left even after the annealing process. Since an oxide layer represents an additional barrier for the field emitted electrons, ${ }^{29,34}$ the removal of this layer would undoubtedly be detected as an increase in the field emission current. A much more stable field emission current would usually be obtained after such treatment, which is also the signature of a cleaner emitter. Another hypothesis is that the high-current treatment could induce an atomic rearrangement at the tip apex. Heating the tip apex in presence of an electric field can lead to the formation of a sharp asperity; $;^{37,43-45}$ the buildup of such a protrusion could then explain the observed change in field emission characteristics.

\section{Characterization of the tips at the atomic level using FIM}

Once field emission measurements have confirmed that a particular W tip is a suitable candidate for FIM studies, this tip is transferred from the preparation chamber of our UHV system into the FIM-STM-AFM chamber for further analysis. The purpose of imaging the tip apex with FIM is to provide us with a better understanding of its atomic structure and with the possibility of engineering it in a configuration which is optimal for SPM experiments.

To perform FIM, we introduce ultrapure helium gas in our UHV chamber and positively bias the tip to a few kilovolts. Gas atoms will be polarized and thus attracted to the tip; through multiple collisions with the tip surface, they will approach thermal equilibrium with the tip. ${ }^{5,46,47}$ When a gas atom is at a critical distance from the tip, there is a high probability of an electron tunneling from the gas atom to the tip. ${ }^{5}$ The newly produced helium ion is then strongly repelled from the tip and accelerated away from it along the field lines. Since a phosphor screen collects the helium ions repelled from the tip, we obtain an image of the tip apex. The intensity of a point on the image corresponds to the probability of field ionization at the corresponding point on the tip. The field ionization probability is a sensitive function of the local electric field, so the brightest features on the image correspond to protruding atoms, since the electric field is enhanced above them. $., 7,15,46$ 

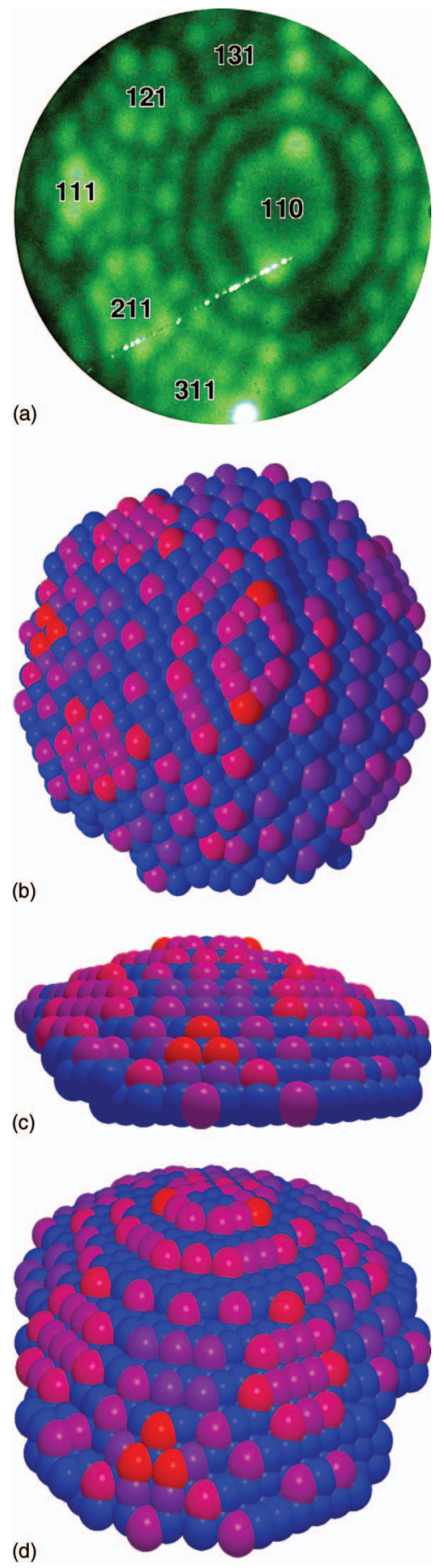

FIG. 2. (Color) (a) Indexed FIM picture of a polycrystalline W tip acquired at $140 \mathrm{~K}$, at $V_{\mathrm{BI}}$ of $4.9 \mathrm{kV}$. (Note that the bright line in the bottom left corner of all our FIM images is due to a crack in our screen assembly.) Images (b), (c), and (d) show the threedimensional, atom-by-atom reconstruction of the apex obtained from the FIM picture in (a). The different shades displayed on this reconstruction reflect the brightness of each atom on the FIM image. In (b), a top view of the apex is presented, which shows a direct correspondence to the FIM picture. Image (c) represents a side view of the tip, with the (111) plane facing the reader. Image (d) shows the apex from an angle, highlighting the spheroidallooking shape of the tip.
It is possible to use these FIM images to reconstruct threedimensional models of the tip apex, as shown in Fig. 2 where a polycrystalline $\mathrm{W}$ tip is presented. To obtain these models, we use a computer program ${ }^{48}$ that allows the user to edit a database of atomic positions in space; the available atomic sites are those of a bcc crystal structure with the lattice parameters of $\mathrm{W}$. These models allow a full characterization of the atomic structure of the apex; this information offers invaluable input to theoretical modeling of the tips for comparison with our results. The knowledge of the surface structure of the tip can also provide useful information about the pressure distribution on the substrate when combined with the force measurements from our AFM.

From a three-dimensional reconstruction, it is possible to calculate the local radius of curvature between two planes; the results can then be compared to those obtained with the popular "ring counting method" which is illustrated in Fig. 3. 5,15,47 Calculating the local radius of curvature between two planes from the three-dimensional reconstruction performed in Fig. 2 is done as follows. By inspection, the center point of the reference plane is determined and, using the computer program, a normal to that plane passing through its center is dropped; the same is done for the neighboring plane and the point where these two normals intersect can then be calculated. The radius is obtained by computing the distance between the intersection point and the top of each plane. For example, applying this technique to the (110) and (111) planes of the reconstruction shown in Fig. 2, one finds that the distance between the intersection point and the top of the (110) plane is $6.0 \mathrm{~nm}$, and the distance between the intersection point and the top of the (111) plane is $5.5 \mathrm{~nm}$. That these two values differ from one another indicates that the surface between these two planes is not spherically shaped; talking about a "radius" becomes less relevant in this case. This points out a clear limitation of the ring counting method, since it assumes that the surface between two neighboring planes can actually be described by an arc of a circle. Note that for the FIM image shown in Fig. 2, the ring counting method yields a local radius of $7.3 \mathrm{~nm}$ between the (110) and the (111) planes, which is larger than both values obtained from the reconstruction.

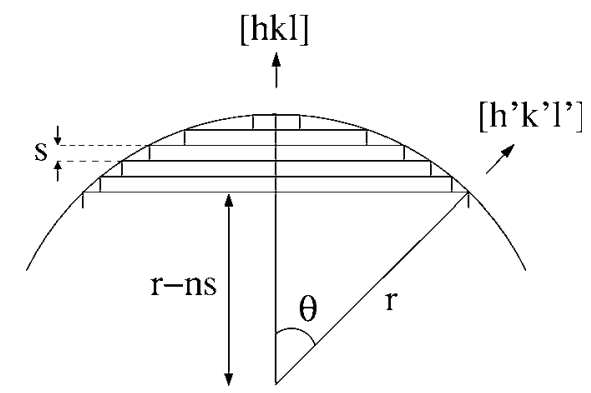

FIG. 3. Illustration of the "ring counting method" used to determine the local radius of curvature $r$ between a reference crystallographic plane of Miller indices $[h k l]$ and a neighboring plane of Miller indices $\left[h^{\prime} k^{\prime} l^{\prime}\right]$ separated by an angle $\theta$. According to the simple geometrical considerations shown on this diagram, the local radius can be found by counting on the FIM image the number of rings $n$ of step height $s$ which can be seen between the centers of the two poles. 
Another limitation of the ring counting method is that there is not always a ring belonging to the reference plane which passes exactly through the center of the neighboring plane; this greatly increases the error on the obtained radius value. From the three-dimensional reconstruction however, the spatial coordinates of the center of any plane can be easily determined; the analysis of the reconstruction then allows the calculation of a more refined radius value than that which is found from the ring counting method.

We can conclude that the radius of curvature varies continuously across the apex surface, even between two adjacent crystallographic planes; departures from the spherical approximation will most likely become more apparent for sharper tips. Therefore, the most accurate and reliable characterization of the tip apex is obtained through a threedimensional, atom-by-atom reconstruction.

Another useful application of FIM is the possibility of engineering the atomic structure of the apex through field evaporation. ${ }^{7,34}$ Field evaporation can lead to a controlled removal of individual $\mathrm{W}$ atoms from the tip; a stable apex terminating in very few atoms can then be achieved. We have successfully applied this technique to a (111) oriented W tip; an example of such atomic manipulation of the apex by controlled field evaporation is presented in Fig. 4(a), where the field evaporation steps reduce the number of atoms on the topmost layer of the apex from 5 to 3 . The resulting (111) trimer is the sharpest stable apex we observed. We did not manage to engineer an apex terminating in a single atom since the trimer field evaporated as a single unit; such simultaneous field evaporation of the three remaining atoms on the apex had also been reported before. ${ }^{7}$ If the trimer is accidentally field evaporated, it is possible to resume the evaporation process on the freshly exposed (111) layer in order to recover a sharp apex. This is illustrated in Fig. 4(b), where another sequence of field evaporation steps for the same tip is shown. This time, the order in which the apex atoms are field evaporated is different than that which is observed in Fig. 4(a). Since the process of field evaporation is statistical in nature, it is very difficult to predict the exact order of the evaporation sequence for more than a few steps; however, it is almost always possible to know which atom will field evaporate next. As illustrated in Fig. 4, it is the brightest edge atom of the apex which disappears in between each picture because the electric field is most enhanced above this atom. This allows incredible control over the final number of atoms on the tip apex. It also corroborates the fact that the trimer evaporates as a single unit, since the three atoms are always equally bright. Note also that because field evaporation is thermally activated, ${ }^{5,15,16}$ the field evaporation rate will depend notably on the vibrational amplitude of the surface atoms; controlled removal of single atoms will thus be much easier at low temperatures, due to the higher stability of the atomic structure above $V_{\mathrm{BI}}$. For tips imaged at room temperature, we repeatedly observed that a slight increase of the voltage above its $V_{\mathrm{BI}}$ value would cause several atoms to field evaporate.

Since planes with a higher work function have a slightly lower evaporation field, $,, 15,16$ and since these planes are closely packed and thus have a larger inter-planar spacing which enhances the field at their edges, atoms belonging to (a)
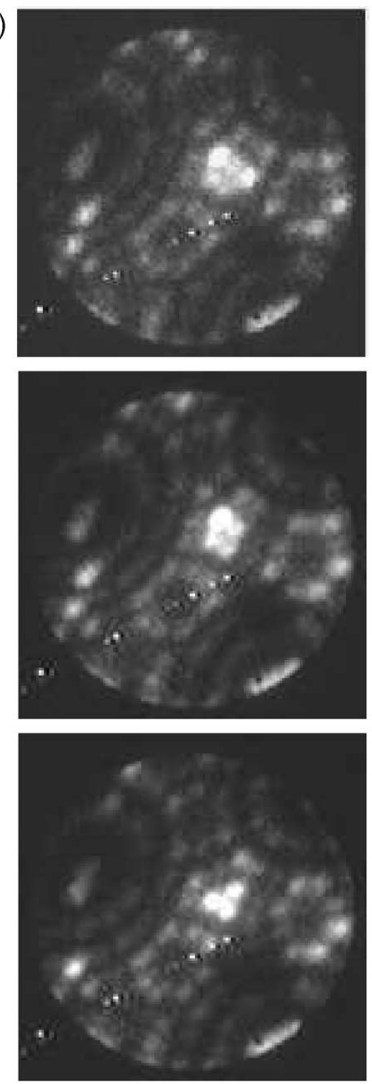

(b)
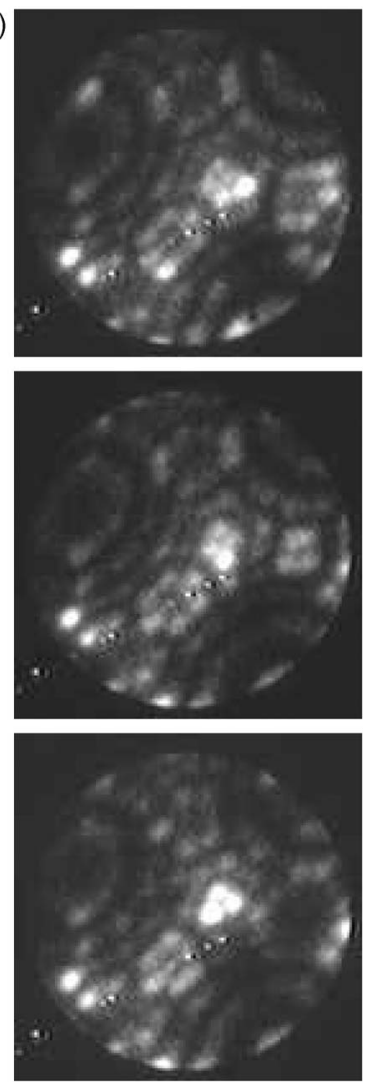

FIG. 4. (a) Sequence of three FIM images of a (111)-oriented W tip showing atomic engineering of its apex by successive field evaporation of single atoms. The images were acquired at $140 \mathrm{~K}$, at $V_{\mathrm{BI}}$ of $5.9 \mathrm{kV}$. In between each picture, an atom from the topmost (111) plane was field evaporated by momentarily increasing the voltage to $6.2 \mathrm{kV}$. (b) FIM images showing another sequence of field evaporation steps for the tip presented in (a); note that the order in which the apex atoms are field evaporated is different in this case.

these planes will be preferentially evaporated at lower voltages. This explains why the $\{110\}$ planes, which have the highest work function $\left(\phi_{110}=5.25 \mathrm{eV}\right)$ for $\mathrm{W}$ and a large step height of $2.23 \AA, 5$ always develop into larger, more open facets on all our FIM images taken at $V_{\mathrm{BI}}$ (see Fig. 2). Such an apex would most likely not yield atomic resolution when scanned over a surface. These planes are quite difficult to engineer in a controlled fashion, due to the lower energy required to field evaporate the atoms. The onset of field evaporation tends to happen at a voltage too close to $V_{\mathrm{BI}}$ to allow field evaporation of single atoms; if the voltage is slightly raised above $V_{\mathrm{BI}}$, an entire layer of (110) atoms quickly field evaporates. It has been our experience that any apex configuration obtained on a (110) plane is not stable for more than a few minutes at $V_{\mathrm{BI}}$. The (111) orientation, with a lower work function $\phi_{111}=4.47 \mathrm{eV}$, a more loosely packed structure, and a smaller interplanar spacing $[s=0.91 \AA$ (Ref. $5)$ ], is much easier to engineer. Since the gap between the $V_{\mathrm{BI}}$ value and the field evaporation voltage is greater for the (111) plane than it is for the (110) plane, the (111) trimer remains very stable at $V_{\mathrm{BI}}$. 
Another advantage of the (111) trimer is its high stability against surface diffusion; the diffusion rate of single atoms on the (111) plane is basically negligible even at room temperature, ${ }^{15,49}$ since the activation energy for single atom self-diffusion is $\sim 1.8 \mathrm{eV}$ for the $\mathrm{W}$ (111) plane. ${ }^{7}$ Atoms on the (110) plane are much more mobile and surface diffusion on this plane is quite rapid; the activation energy for surface diffusion on the (110) plane is about $\sim 0.9 \mathrm{eV} .{ }^{5}$ In order to minimize the movement of apex atoms while scanning over a surface or performing STM spectroscopy measurements, a (111) trimer should thus be chosen over a (110) apex configuration. Moreover, a single-atom tip could be obtained by vapor deposition of an atom onto the (111) trimer; this technique has already been successfully developed by Fink. ${ }^{7}$ Due to the loosely packed structure of the trimer and the high activation energy for surface diffusion on the (111) plane, the vapor-deposited atom creates a very stable protrusion. ${ }^{15}$ This single-atom protrusion could be made of a different metal than the underlying tungsten tip, and could even have a lower evaporation field (bulk property) than the $F_{\mathrm{BI}}$ of helium, as was experimentally demonstrated by the imaging of gold atoms deposited on a (111) W tip. ${ }^{34}$

\section{Limitations of FIM when applied to very sharp tips}

Since FIM is a point projection microscope, the magnification is inversely proportional to the tip radius. ${ }^{47}$ This becomes an important limitation for very sharp tips because only a few atoms can be seen on the FIM image. Since crystallographic symmetries are difficult to recognize, the interpretation of the image and the reconstruction of the tip become almost impossible; this is illustrated in Fig. 5, where a highly magnified FIM image of an extremely sharp polycrystalline $\mathrm{W}$ tip is presented. One can see that the apex of this tip terminates in a single atom, but a more complete description of the atomic structure of the apex cannot be deduced from this FIM picture. This observation implies that small radius tips which would be perfectly suitable for STM imaging and spectroscopy have an atomic structure that cannot be fully characterized through FIM imaging.

One could exploit the phenomenon of field evaporation in order to decipher the structure of a highly magnified tip. By carefully evaporating away successive atomic layers, the tip becomes blunt, thus reducing the magnification. If FIM pictures are taken after every field evaporation step, in principle it should be possible to reconstruct the original structure of the tip from its underlying structure. This idea is illustrated in Fig. 6, where field evaporation is performed on a very sharp tip [Fig. 6(a)], until a blunter tip with larger facets and recognizable symmetries is obtained [Fig. 6(b)]. One would then use the FIM image presented in Fig. 6(b) to perform a three-dimensional reconstruction of the tip, and the atomic layers corresponding to the intermediate FIM images taken during the field evaporation process could be successively added to this reconstruction until one achieves the structure of the sharp tip in Fig. 6(a). This process would undoubtedly be very tedious, and it is most likely that not all the evaporation steps would be recorded on camera because atoms would keep on field evaporating during the exposure time

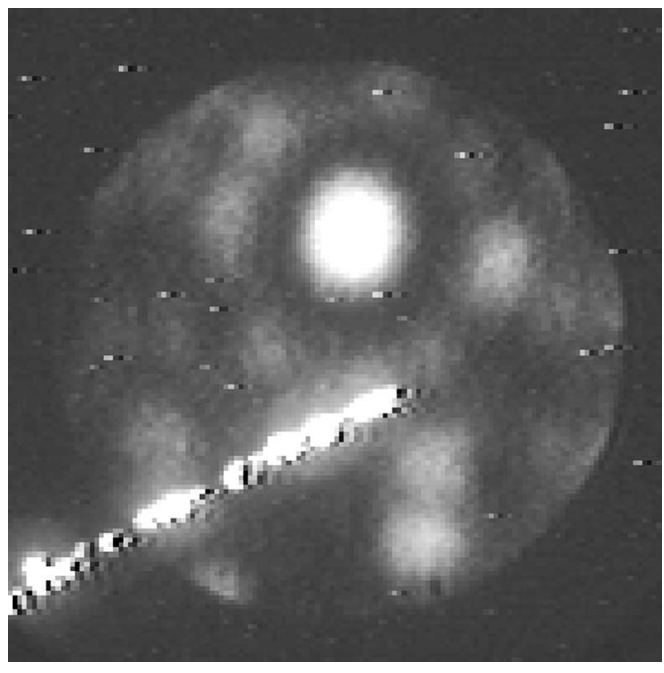

FIG. 5. FIM picture of a polycrystalline $\mathrm{W}$ tip which is so sharp that only a few atoms are imaged; the ring counting method and the three-dimensional reconstruction are thus impossible to apply for this tip apex from this FIM picture alone. This FIM image was acquired at room temperature, at $V_{\mathrm{BI}}$ of $2.4 \mathrm{kV}$. Note that this single-atom tip was not obtained through controlled field evaporation of the apex; it just happened to be caught on camera. As mentioned before, a particular apex configuration on a (110) plane is not stable and this can be seen by comparing this image with Fig. 6(a), which shows the same tip but this time terminating in two atoms; both images were acquired at the same $V_{\mathrm{BI}}$ and only a few seconds apart.

often required to get a clear FIM picture, thus increasing the error on the final reconstruction. To overcome this technical limitation, a three-dimensional atom probe field ion microscope could be used to map the position of each atom on the tip from their time of flight when field evaporated from the tip. A three-dimensional picture of any sharp and highly magnified tip could thus be obtained. However, this would lead to a substantial complication in a combined FIM-SPM setup as one would need to incorporate a time-of-flight spectrometer, as well as pulsing electrodes. ${ }^{50}$ The downside of any technique using field evaporation to reconstruct the (a)

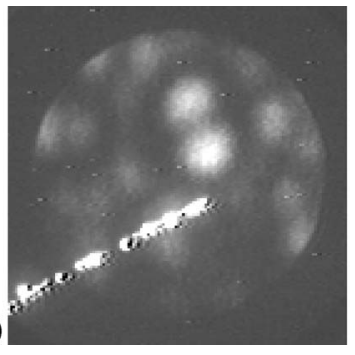

(b)

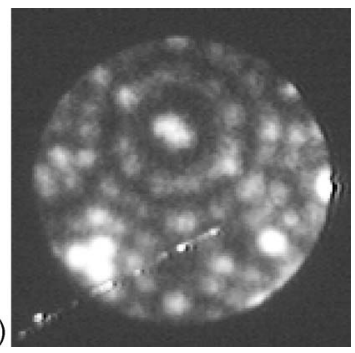

FIG. 6. FIM images of a polycrystalline W tip. Image (a) shows the tip when it was still very sharp; it was acquired at a low $V_{\mathrm{BI}}$ of $2.4 \mathrm{kV}$ and displays a high magnification. Image (b) shows the same tip after it got blunt by field evaporation; the increase in tip radius is confirmed by the higher $V_{\mathrm{BI}}$ of $4.0 \mathrm{kV}$ and the obvious decrease in magnification. Note that the left image was taken at room $T$ and the right image at $140 \mathrm{~K}$; the temperature does not affect the magnification. 
atomic structure of a tip is that it is an irreversible and destructive process for the tip.

\section{CONCLUSIONS}

A multistage tip preparation and characterization technique was studied with the aim of fabricating atomically characterized $\mathrm{W}$ tips which can be used for well-defined SPM experiments. Using this technique, sharp polycrystalline $\mathrm{W}$ tips with apex radii of a few nanometers can reproducibly be obtained; furthermore, a single-crystal, (111)oriented W tip can be engineered into a stable apex configuration terminating in three atoms. The (111) trimer is a good configuration for a tip in various SPM studies. It is easy to engineer due to its lattice structure and it is very stable against surface diffusion. It also provides a template on which a single atom of a different chemical specie can be vapor deposited. Moreover, its striking threefold symmetry can be easily recognized even on highly magnified FIM images. This magnification issue is the major drawback of FIM techniques; only tips with a large enough radius can be atomically characterized through a three-dimensional reconstruction of their apex. Using tips engineered and characterized with FIM, atomically defined SPM experiments can be performed and will hopefully lead to a much better agreement between experimental results and theoretical predictions.

\section{ACKNOWLEDGMENTS}

We wish to thank Mark Orchard-Webb for his help with the three-dimensional atom-by-atom reconstruction of our tips from our FIM images, and Robert Gagnon for his assistance in acquiring the scanning electron microscopy images necessary for the characterization of our tips. We would also like to acknowledge support from NSERC, NATEQ, CIAR, IBM and NanoQuébec.
*Electronic address: grutter@physics.mcgill.ca

${ }^{1}$ W. A. Hofer, A. S. Foster, and A. L. Shluger, Rev. Mod. Phys. 75, 1287 (2003).

${ }^{2}$ H. Hug, R. Bennewitz, and E. Meyer, Scanning Probe Microscopy: The Lab on a Tip (Springer-Verlag, New York, 2003).

${ }^{3}$ Noncontact Atomic Force Microscopy, edited by S. Morita, R. Wiesendanger, and E. Meyer (Springer-Verlag, New York, 2002).

${ }^{4}$ Z. J. Donhauser et al., Science 292, 2303 (2001).

${ }^{5}$ T. T. Tsong, Atom-Probe Field Ion Microscopy (Cambridge University Press, New York, 1990).

${ }^{6}$ Y. Kuk and P. J. Silverman, Appl. Phys. Lett. 48, 1597 (1986).

${ }^{7}$ H.-W. Fink, IBM J. Res. Dev. 30, 460 (1986).

${ }^{8}$ G. Cross, A. Schirmeisen, A. Stalder, P. Grütter, M. Tschudy, and U. Dürig, Phys. Rev. Lett. 80, 4685 (1998).

${ }^{9}$ A. Schirmeisen, G. Cross, A. Stalder, P. Grütter, and U. Dürig, New J. Phys. 2, 29 (2000).

${ }^{10}$ Y. Sun, H. Mortensen, S. Schär, A.-S. Lucier, Y. Miyahara, P. Grütter, and W. Hofer, Phys. Rev. B 71, 193407 (2005).

${ }^{11}$ A. Stalder, Ph.D. thesis, University of Freiburg, 1995.

${ }^{12}$ T. Hashizume, Y. Hasegawa, I. Kamiya, T. Ide, I. Sumita, S. Hyodo, T. Sakurai, H. Tochihara, M. Kubota, and Y. Murata, J. Vac. Sci. Technol. A 8, 233 (1990).

${ }^{13}$ T. Sakurai, T. Hashizume, I. Kamiya, Y. Hasegawa, T. Ide, M. Miyao, I. Sumita, A. Sakai, and S. Hyodo, J. Vac. Sci. Technol. A 7, 1684 (1989).

${ }^{14}$ S. A. Smallwood, Ph.D. thesis, University of Maine, 1992.

${ }^{15}$ M. K. Miller, A. Cerezo, M. G. Hetherington, and G. D. W. Smith, Atom Probe Field Ion Microscopy (Oxford University Press, New York, 1996).

${ }^{16}$ K. M. Bowkett and D. A. Smith, Field-Ion Microscopy, Defects in Crystalline Solids Vol. 2 (North-Holland Publishing Company, Amsterdam, 1970).

${ }^{17}$ L. E. Murr, Electron and Ion Microscopy and Microanalysis: Principles and Applications, 2nd ed. (Marcel Dekker, New York, 1991).
${ }^{18}$ A. J. Melmed, J. Vac. Sci. Technol. B 9, 601 (1991).

${ }^{19}$ H. Lemke, T. Göddenhenrich, H. P. Bochem, U. Hartmann, and C. Heiden, Rev. Sci. Instrum. 61, 2538 (1990).

${ }^{20}$ J. P. Ibe, P. P. BeyJr., S. L. Brandow, R. A. Brizzolara, N. A. Burnham, D. P. DiLella, K. P. Lee, C. R. K. Marrian, and R. J. Colton, J. Vac. Sci. Technol. A 8, 3570 (1990).

${ }^{21}$ U. J. Quaade and L. Oddershede, Europhys. Lett. 57, 611 (2002).

${ }^{22}$ R. Nicolaides et al., J. Vac. Sci. Technol. A 6, 445 (1988).

${ }^{23}$ R. Zhang and D. G. Ivey, J. Vac. Sci. Technol. B 14, 1 (1996).

${ }^{24}$ Y. Sun, Ph.D. thesis, McGill University, 2004.

${ }^{25}$ J. Garnaes, F. Kragh, K. A. Mørch, and A. R. Thölén, J. Vac. Sci. Technol. A 8, 441 (1990).

${ }^{26}$ E. Paparazzo, L. Moretto, S. Selci, M. Righini, and I. Farné, Vacuum 52, 421 (1999).

${ }^{27}$ A. I. Oliva, A. Romero G., J. L. Peña, E. Anguiano, and M. Aguilar, Rev. Sci. Instrum. 67, 1917 (1996).

${ }^{28}$ L. Ottaviano, L. Lozzi, and S. Santucci, Rev. Sci. Instrum. 74, 3368 (2003).

${ }^{29}$ I. Ekvall, E. Wahlström, D. Claesson, H. Olin, and E. Olsson, Meas. Sci. Technol. 10, 11 (1999).

${ }^{30}$ A. Cricenti, E. Paparazzo, M. A. Scarselli, L. Moretto, and S. Selci, Rev. Sci. Instrum. 65, 1558 (1994).

${ }^{31}$ C. J. Chen, Introduction to Scanning Tunneling Microscopy (Oxford University Press, New York, 1993).

${ }^{32}$ S. W. H. Yih and C. T. Wang, Tungsten: Sources, Metallurgy, Properties, and Applications (Plenum Press, New York, 1979).

${ }^{33}$ K. C. Li and C. Y. Wang, Tungsten: Its History, Geology, OreDressing, Metallurgy, Chemistry, Analysis, Applications, and Economics, 3rd ed. (Reinhold Publishing Corporation, New York, 1955).

${ }^{34}$ A. Schirmeisen, Ph.D. thesis, McGill University, 1999.

${ }^{35}$ H. S. Kim, M. L. Yu, U. Staufer, L. P. Murray, D. P. Kern, and T. H. P. Chang, J. Vac. Sci. Technol. B 11, 2327 (1993).

${ }^{36}$ V. T. Binh, A. Piquet, H. Roux, R. Uzan, and M. Drechsler, J. Phys. E 9, 377 (1976).

${ }^{37}$ U. Staufer, L. P. Muray, D. P. Kern, and T. H. P. Chang, J. Vac. 
Sci. Technol. B 9, 2962 (1991).

${ }^{38}$ R. H. Fowler and L. Nordheim, Proc. R. Soc. London, Ser. A 119, 173 (1928).

${ }^{39}$ R. Gomer, Field Emission and Field Ionization, Harvard Monographs in Applied Science Vol. 9 (Harvard University Press, Cambridge, 1961).

${ }^{40}$ J. Méndez, M. Luna, and A. M. Baró, Surf. Sci. 266, 294 (1992).

${ }^{41}$ W. Qian, M. R. Scheinfein, and J. C. H. Spence, J. Appl. Phys. 73, 7041 (1993).

${ }^{42}$ M. P. Marder, Condensed Matter Physics (Wiley-Interscience, New York, 2000).

${ }^{43}$ J. A. Meyer, S. J. Stranick, J. B. Wang, and P. S. Weiss, Ultramicroscopy 42-44, 1538 (1992).
${ }^{44}$ V. T. Binh and J. Marien, Surf. Sci. Lett. 202, L539 (1988).

${ }^{45}$ V. T. Binh, J. Microsc. 152, 355 (1988).

${ }^{46}$ T. Sakurai, A. Sakai, and H. W. Pickering, Atom-Probe Field Ion Microscopy and Its Applications, Advances in Electronics and Electron Physics (Academic Press, Boston, 1989).

${ }^{47}$ J. A. Panitz, J. Phys. E 15, 1281 (1982).

${ }^{48}$ M. Orchard-Webb, Computer code for 3D tip reconstruction (McGill University, Montreal, Canada, 2003).

${ }^{49}$ O. Nishikawa, M. Tomitori, and F. Katsuki, J. Microsc. 152, 637 (1988).

${ }^{50}$ A. Cerezo, T. J. Godfrey, and G. D. W. Smith, Rev. Sci. Instrum. 59, 862 (1988). 